\title{
FIRMLY NONEXPANSIVE MAPPINGS IN CLASSES OF GEODESIC SPACES
}

\author{
DAVID ARIZA-RUIZ, LAURENŢIU LEUŞTEAN, AND GENARO LÓPEZ-ACEDO
}

\begin{abstract}
Firmly nonexpansive mappings play an important role in metric fixed point theory and optimization due to their correspondence with maximal monotone operators. In this paper we do a thorough study of fixed point theory and the asymptotic behaviour of Picard iterates of these mappings in different classes of geodesic spaces, such as (uniformly convex) $W$-hyperbolic spaces, Busemann spaces and CAT(0) spaces. Furthermore, we apply methods of proof mining to obtain effective rates of asymptotic regularity for the Picard iterations.
\end{abstract}

\section{INTRODUCTION}

Let $C$ be a closed convex subset of a Hilbert space $H$. Firmly contractive mappings were defined by Browder [5] as mappings $T: C \rightarrow H$ satisfying the following inequality for all $x, y \in C$ :

$$
\|T x-T y\|^{2} \leq\langle x-y, T x-T y\rangle .
$$

As Browder points out, these mappings play an important role in the study of (weak) convergence for sequences of nonlinear operators. An example of a firmly contractive mapping is the metric projection $P_{C}: H \rightarrow H$, defined by $P_{C}(x)=$ $\operatorname{argmin}_{y \in C}\{\|x-y\|\}$. One can easily see that any firmly contractive mapping $T$ is nonexpansive, i.e. satisfies $\|T x-T y\| \leq\|x-y\|$ for all $x, y \in C$. The converse is not true, as one can see by taking $T=-I d$.

In his study of nonexpansive projections on subsets of Banach spaces, Bruck [6] defined a firmly nonexpansive mapping $T: C \rightarrow E$, where $C$ is a closed convex subset of a real Banach space $E$, to be a mapping with the property that for all $x, y \in C$ and $t \geq 0$,

$$
\|T x-T y\| \leq\|(1-t)(T x-T y)+t(x-y)\| .
$$

In Hilbert spaces these mappings coincide with the firmly contractive ones introduced by Browder. As Bruck shows, to any nonexpansive selfmapping $T: C \rightarrow C$ that has fixed points, one can associate a 'large' family of firmly nonexpansive mappings having the same fixed point set with $T$. Hence, from the point of view of the existence of fixed points on convex closed sets, firmly nonexpansive mappings exhibit a similar behaviour with the nonexpansive ones. However, this is no

Received by the editors March 10, 2012 and, in revised form, May 29, 2012 and September 22, 2012 .

2010 Mathematics Subject Classification. Primary 47H09, 47H10, 53C22; Secondary 03F10, 47H05, 90C25, 52A 41 .

Key words and phrases. Firmly nonexpansive mappings, geodesic spaces, uniform convexity, Picard iterates, asymptotic regularity, $\Delta$-convergence, proof mining, effective bounds, minimization problems. 
longer true if we consider nonconvex domains [43. Firmly nonexpansive mappings in Banach spaces have also been studied in [7] and [39].

If $T$ is firmly nonexpansive and has fixed points, it is well known [5] that the Picard iterate $\left(T^{n} x\right)$ converges weakly to a fixed point of $T$ for any starting point $x$, while this is not true for nonexpansive mappings (take again $T=-I d$ ). This is a first reason for the importance of firmly nonexpansive mappings.

A second reason for the importance of this class of mappings is their correspondence with maximal monotone operators, due to Minty 34.

The resolvent of a monotone operator was introduced by Minty [34] in Hilbert spaces and by Brézis, Crandall and Pazy [3] in Banach spaces. Among other applications, the resolvent has proved to be very useful in the study of the asymptotic behaviour of the solutions of the Cauchy abstract problem governed by a monotone operator; see for instance [16, 36, 47]. Given a maximal monotone operator $A: H \rightarrow 2^{H}$ and $\mu>0$, its associated resolvent of order $\mu$, defined by $J_{\mu}^{A}:=(I d+\mu A)^{-1}$, is a firmly nonexpansive mapping from $H$ to $H$ and the set of fixed points of $J_{\mu}^{A}$ coincides with the set of zeros of $A$. We refer to [2] for a very nice presentation of this correspondence. Rockafellar's [42] proximal point algorithm uses the resolvent to approximate the zeros of maximal monotone operators.

The subdifferential of a proper, convex and lower semicontinuous function $F$ : $H \rightarrow(-\infty, \infty]$ is a maximal monotone operator. Hence the resolvent associated to the subdifferential is a firmly nonexpansive mapping that coincides with the proximal map introduced by Moreau [35. The proximal point algorithm for approximating the minimizers of $F$ is based on the weak convergence towards a fixed point of the Picard iterate of the resolvent and the fact that the minimizers of $F$ are the fixed points of the resolvent.

During the last 20 years there has been a fruitful direction of research that consists of extending techniques and results obtained in normed spaces to metric spaces without linear structure. For instance, minimization problems associated to convex functionals have been solved in the setting of Riemannian manifolds 15, 31, while some problems have been modelled as abstract Cauchy equations in the framework of nonpositive curvature geodesic metric spaces (see [33, 45] and the references therein). Although apparently the framework and the conceptual approach in the previous problems are quite different, it is possible, as in the case of normed spaces, to find a bridge between them through firmly nonexpansive mappings.

The goal of our work is twofold. First we generalize known results on firmly nonexpansive mappings in Hilbert or Banach spaces to suitable classes of geodesic spaces. Second we obtain effective results on the asymptotic behaviour of Picard iterations.

In Section 2 we give basic definitions and properties of the classes of geodesic spaces we consider in this paper: $W$-hyperbolic spaces, $U C W$-hyperbolic spaces, Busemann spaces and CAT(0) spaces. We recall properties of asymptotic centers in such spaces that are essential for our results.

Firmly nonexpansive mappings in the Hilbert ball and, more generally, in hyperbolic spaces, have already been studied in [18,40,41 and, more recently, in the paper by Kopecká and Reich [27. In Section 3 we extend Bruck's definition of a firmly nonexpansive mapping to our class of $W$-hyperbolic spaces. We show that, in the setting of $\mathrm{CAT}(0)$ spaces, the metric projection on a closed convex set and 
the resolvent of a proper, convex and lower semicontinuous mapping are firmly nonexpansive. Furthermore, Bruck's association of a family of firmly nonexpansive mappings to any nonexpansive mapping is adapted to Busemann spaces.

Section 4 contains a fixed point theorem for firmly nonexpansive mappings defined on finite unions of closed convex subsets of a complete $U C W$-hyperbolic space. Our result generalizes and strengthens Smarzewski's 43 fixed point theorem for uniformly convex Banach spaces. In this section we also obtain new results about periodic points of (firmly) nonexpansive mappings.

In the next section we study the asymptotic behaviour of Picard iterates of firmly nonexpansive mappings, extending to $W$-hyperbolic spaces the results of Reich and Shafrir [40,41. As a consequence, we obtain that any firmly nonexpansive mapping with bounded orbits is asymptotically regular.

A concept of weak convergence in geodesic spaces is the so-called $\Delta$-convergence, defined by Lim 32. Applying our asymptotic regularity result and general properties of Fejér monotone sequences, we prove in Section 6, in the setting of complete $U C W$-hyperbolic spaces, the $\Delta$-convergence of Picard iterates of a firmly nonexpansive mapping to a fixed point. As a consequence, one gets the $\Delta$-convergence of a proximal point like algorithm to a minimizer of a proper, convex and lower semicontinuous mapping defined on a $\mathrm{CAT}(0)$ space.

In the final section of the paper we obtain effective rates of asymptotic regularity for Picard iterations, applying methods of proof mining, similar to the ones used for Krasnoselski-Mann iterations of nonexpansive mappings by Kohlenbach [23] in Banach spaces and the second author [29] in $U C W$-hyperbolic spaces. We point out that our results are new even for uniformly convex Banach spaces. In the case of CAT(0) spaces we obtain a quadratic rate of asymptotic regularity. Proof mining is a paradigm of research concerned with the extraction, using tools from mathematical logic, of hidden finitary and combinatorial content, such as algorithms and effective bounds, from proofs that make use of highly infinitary principles. We refer to Kohlenbach's book [25] for details.

\section{Classes of Geodesic spaces - Definitions ANd PROperties}

A $W$-hyperbolic space $(X, d, W)$ is a metric space $(X, d)$ together with a convexity mapping $W: X \times X \times[0,1] \rightarrow X$ satisfying

$$
\begin{gathered}
d(z, W(x, y, \lambda)) \leq(1-\lambda) d(z, x)+\lambda d(z, y), \\
d(W(x, y, \lambda), W(x, y, \tilde{\lambda}))=|\lambda-\tilde{\lambda}| \cdot d(x, y), \\
W(x, y, \lambda)=W(y, x, 1-\lambda), \\
d(W(x, z, \lambda), W(y, w, \lambda)) \leq(1-\lambda) d(x, y)+\lambda d(z, w) .
\end{gathered}
$$

The convexity mapping $W$ was first considered by Takahashi in [46, where a triple $(X, d, W)$ satisfying (W1) is called a convex metric space. $W$-hyperbolic spaces were introduced by Kohlenbach [24, and we refer to [25, p. 384] for a comparison between them and other notions of 'hyperbolic space' that can be found in the literature (see for example [17,21,41]). The class of $W$-hyperbolic spaces includes (convex subsets of) normed spaces, the Hilbert ball (see [18] for a book treatment), as well as $\mathrm{CAT}(0)$ spaces 4 . 
We shall denote a $W$-hyperbolic space simply by $X$ when the metric $d$ and the mapping $W$ are clear from the context. One can easily see that

$$
d(x, W(x, y, \lambda))=\lambda d(x, y) \quad \text { and } \quad d(y, W(x, y, \lambda))=(1-\lambda) d(x, y) .
$$

Furthermore, $W(x, y, 0)=x, W(x, y, 1)=y$ and $W(x, x, \lambda)=x$.

Let us recall now some notions concerning geodesics. Let $(X, d)$ be a metric space. A geodesic path in $X$ (geodesic in $X$ for short) is a map $\gamma:[a, b] \rightarrow X$ satisfying

$$
d(\gamma(s), \gamma(t))=|s-t| \text { for all } s, t \in[a, b] .
$$

A geodesic segment in $X$ is the image of a geodesic in $X$. If $\gamma:[a, b] \rightarrow X$ is a geodesic in $X, \gamma(a)=x$ and $\gamma(b)=y$, we say that the geodesic $\gamma$ joins $x$ and $y$ or that the geodesic segment $\gamma([a, b])$ joins $x$ and $y ; x$ and $y$ are also called the endpoints of $\gamma$.

A metric space $(X, d)$ is said to be a (uniquely) geodesic space if every two distinct points are joined by a (unique) geodesic segment.

If $\gamma([a, b])$ is a geodesic segment joining $x$ and $y$ and $\lambda \in[0,1], z:=\gamma((1-\lambda) a+\lambda b)$ is the unique point in $\gamma([a, b])$ satisfying

$$
d(z, x)=\lambda d(x, y) \quad \text { and } \quad d(z, y)=(1-\lambda) d(x, y) .
$$

In the sequel, we shall use the notation $[x, y]$ for the geodesic segment $\gamma([a, b])$ and we shall denote this $z$ by $(1-\lambda) x \oplus \lambda y$, provided that there is no possible ambiguity.

Given three points $x, y, z$ in a metric space $(X, d)$, we say that $y$ lies between $x$ and $z$ if these points are pairwise distinct and if we have $d(x, z)=d(x, y)+$ $d(y, z)$. Obviously, if $y$ lies between $x$ and $z$, then $y$ also lies between $z$ and $x$. Furthermore, the relation of betweenness also satisfies a transitivity property (see, e.g., [38, Proposition 2.2.13]):

Proposition 2.1. Let $X$ be a metric space and $x, y, z, w$ be pairwise distinct points of $X$. The following statements are equivalent:

(i) $y$ lies between $x$ and $z$ and $z$ lies between $x$ and $w$.

(ii) $y$ lies between $x$ and $w$ and $z$ lies between $y$ and $w$.

The following betweenness property expresses another form of 'transitivity', which is not true in general metric spaces:

(6) for all $x, y, z, w \in X$, if $y$ lies between $x$ and $z$ and $z$ lies between $y$ and $w$, then $y$ and $z$ both lie between $x$ and $w$.

By induction one gets

Lemma 2.2. Let $X$ be a metric space satisfying (6). For all $n \geq 2$ and all $x_{0}, x_{1}, \ldots, x_{n} \in X$, we have that

$$
\begin{aligned}
& \text { if for all } k=1, \ldots, n-1, x_{k} \text { lies between } x_{k-1} \text { and } x_{k+1} \text {, } \\
& \text { then for all } k=1, \ldots, n-1, x_{k} \text { lies between } x_{0} \text { and } x_{k+1} \text {. }
\end{aligned}
$$

The next lemma collects some well-known properties of geodesic spaces. We refer to [38] for details.

Lemma 2.3. Let $(X, d)$ be a geodesic space.

(i) For every pairwise distinct points $x, y, z$ in $X, y$ lies between $x$ and $z$ if and only if there exists a geodesic segment $[x, z]$ containing $y$. 
(ii) For every points $x, y, z, w$ and any geodesic segment $[x, y]$, if $z, w \in[x, y]$, then either $d(x, z)+d(z, w)=d(x, w)$ or $d(w, z)+d(z, y)=d(w, y)$.

(iii) For every geodesic segment $[x, y]$ in $X$ and $\lambda, \tilde{\lambda} \in[0,1]$,

$$
d((1-\lambda) x \oplus \lambda y,(1-\tilde{\lambda}) x \oplus \tilde{\lambda} y)=|\lambda-\tilde{\lambda}| d(x, y) .
$$

(iv) Let $\gamma:[a, b] \rightarrow X$ be a geodesic that joins $x$ and $y$. Define

$$
\gamma^{-}:[a, b] \rightarrow X, \quad \gamma^{-}(s)=\gamma(a+b-s) .
$$

Then $\gamma^{-}$is a geodesic that joins $y$ and $x$ such that $\gamma^{-}([a, b])=\gamma([a, b])$.

(v) Let $\gamma, \eta:[a, b] \rightarrow X$ be geodesics. If $\gamma([a, b])=\eta([a, b])$ and $\gamma(a)=\eta(a)$ (or $\gamma(b)=\eta(b))$, then $\gamma=\eta$.

(vi) The following statements are equivalent:

(a) $X$ is uniquely geodesic.

(b) For any $x \neq y \in X$ and any $\lambda \in[0,1]$ there exists a unique element $z \in X$ such that

$$
d(x, z)=\lambda d(x, y) \quad \text { and } \quad d(y, z)=(1-\lambda) d(x, y) .
$$

Lemma 2.4. Let $X$ be a uniquely geodesic space.

(i) For all $x, y \in X,[x, y]=\{(1-\lambda) x \oplus \lambda y \mid \lambda \in[0,1]\}$.

(ii) For every pairwise distinct points $x, y, z$ in $X, y$ lies between $x$ and $z$ if and only if $y \in[x, z]$.

(iii) Let $x, y, z, w$ be pairwise distinct points in $X$ such that $y=(1-\lambda) x \oplus \lambda z$ and $z=(1-\alpha) x \oplus \alpha w$ for some $\lambda, \alpha \in(0,1)$. Then $z=(1-\mu) y \oplus \mu w$, where $\mu=\frac{(1-\lambda) \alpha}{1-\alpha \lambda}$.

Proof. (i) and (ii) are obvious.

(iii) Applying (ii), Lemma 2.3 (ii) and Proposition [2.1, one gets that $z \in[y, w]$. Thus, $z=(1-\mu) y \oplus \mu w$ for some $\mu \in(0,1)$. Furthermore,

$$
\begin{aligned}
d(z, y) & =(1-\lambda) d(x, z)=(1-\lambda) \alpha d(x, w)=\frac{(1-\lambda) \alpha}{1-\alpha} d(z, w) \\
& =\frac{(1-\lambda) \alpha}{1-\alpha} \cdot(1-\mu) d(y, w)=\frac{(1-\lambda) \alpha}{1-\alpha} \cdot \frac{1-\mu}{\mu} d(z, y) .
\end{aligned}
$$

Thus, $\frac{(1-\lambda) \alpha}{1-\alpha} \cdot \frac{1-\mu}{\mu}=1$, and the conclusion follows immediately.

Let $(X, d, W)$ be a $W$-hyperbolic space. For all $x, y \in X$, let us define

$$
[x, y]_{W}:=\{W(x, y, \lambda) \mid \lambda \in[0,1]\} .
$$

Then $[x, x]_{W}=\{x\}$ for all $x \in X$. A subset $C \subseteq X$ is convex if $[x, y]_{W} \subseteq C$ for all $x, y \in C$. Open and closed balls are convex sets. A nice feature of our setting is that any convex subset is itself a $W$-hyperbolic space.

Following [46], we call a $W$-hyperbolic space strictly convex if for any $x \neq y \in X$ and any $\lambda \in(0,1)$ there exists a unique element $z \in X$ (namely $z=W(x, y, \lambda)$ ) such that

$$
d(x, z)=\lambda d(x, y) \quad \text { and } \quad d(y, z)=(1-\lambda) d(x, y) .
$$


Proposition 2.5. Let $(X, d, W)$ be a $W$-hyperbolic space. Then

(i) $X$ is a geodesic space and for all $x \neq y \in X,[x, y]_{W}$ is a geodesic segment joining $x$ and $y$.

(ii) $X$ is a uniquely geodesic space if and only if it is strictly convex.

(iii) If $X$ is uniquely geodesic, then

(a) $W$ is the unique convexity mapping that makes $(X, d, W)$ a $W$-hyperbolic space.

(b) For all $x, y \in X$ and $\lambda \in[0,1], W(x, y, \lambda)=(1-\lambda) x \oplus \lambda y$.

Proof. (i) For $x \neq y \in X$, the map we should have $W_{x y}:[0, d(x, y)] \rightarrow X$,

$$
W_{x y}(\alpha)=W\left(x, y, \frac{\alpha}{d(x, y)}\right) .
$$

is a geodesic satisfying $W_{x y}([0, d(x, y)])=[x, w]_{W}$.

(ii) By Lemma 2.3 vil).

(iii) (b) is obvious. In the sequel we prove (a). Let $W^{\prime}: X \times X \times[0,1] \rightarrow X$ be another convexity mapping such that $\left(X, d, W^{\prime}\right)$ is a $W$-hyperbolic space. For $\lambda \in[0,1]$ and $x \in X$ one has $W(x, x, \lambda)=W^{\prime}(x, x, \lambda)=x$. Let $x, y \in$ $X, x \neq y$. Then $[x, y]_{W}$ and $[x, y]_{W^{\prime}}$ are geodesic segments that join $x$ and $y$. Hence we must have that $[x, y]_{W}=[x, y]_{W^{\prime}}$, that is $W_{x y}([0, d(x, y)])=$ $W_{x y}^{\prime}([0, d(x, y)])$. Since $W_{x y}(0)=W_{x y}^{\prime}(0)=x$, we can apply Lemma2.3( (v) to get that $W_{x y}=W_{x y}^{\prime}$, so that $W(x, y, \lambda)=W^{\prime}(x, y, \lambda)$.

An important class of $W$-hyperbolic spaces are the so-called Busemann spaces, used by Busemann [9, 10, to define a notion of 'nonpositively curved space'. We refer to [38] for an extensive study. Let us recall that a map $\gamma:[a, b] \rightarrow X$ is an affinely reparametrized geodesic if $\gamma$ is a constant path or there exist an interval $[c, d]$ and a geodesic $\gamma^{\prime}:[c, d] \rightarrow X$ such that $\gamma=\gamma^{\prime} \circ \psi$, where $\psi:[a, b] \rightarrow[c, d]$ is the unique affine homeomorphism between the intervals $[a, b]$ and $[c, d]$.

A geodesic space $(X, d)$ is a Busemann space if for any two affinely reparametrized geodesics $\gamma:[a, b] \rightarrow X$ and $\gamma^{\prime}:[c, d] \rightarrow X$, the map

$$
D_{\gamma, \gamma^{\prime}}:[a, b] \times[c, d] \rightarrow \mathbb{R}, \quad D_{\gamma, \gamma^{\prime}}(s, t)=d\left(\gamma(s), \gamma^{\prime}(t)\right)
$$

is convex. Examples of Busemann spaces are strictly convex normed spaces. In fact, a normed space is a Busemann space if and only if it is strictly convex.

Proposition 2.6. Let $(X, d)$ be a metric space. The following two statements are equivalent:

(i) $X$ is a Busemann space.

(ii) There exists a (unique) convexity mapping $W$ such that $(X, d, W)$ is a uniquely geodesic $W$-hyperbolic space.

Proof. $(i) \Rightarrow(i i)$ Assume that $X$ is Busemann. By [38, Proposition 8.1.4], any Busemann space is uniquely geodesic. For any $x, y \in X$, let $[x, y]$ be the unique geodesic segment that joins $x$ and $y$ and define

$$
W: X \times X \times[0,1] \rightarrow X, \quad W(x, y, \lambda)=(1-\lambda) x \oplus \lambda y .
$$

Let us verify (W1)-(W4): (W4) follows from [38, Proposition 8.1.2(ii)]; (W2) follows from Lemma 2.3 (iii); (W1) follows from (W4) applied with $z=x$ and the fact that $W(x, x, \lambda)=x ;(\mathrm{W} 3)$ follows by Lemma 2.3(iv).

$($ ii $) \Rightarrow($ i) Apply [38, Proposition 8.1.2(ii)] and (W4). 
A very useful feature of Busemann spaces is the following (see [38, Proposition 8.2.4])

Lemma 2.7. Every Busemann space satisfies the betweenness property (6). Hence, Lemma 2.2 holds in Busemann spaces.

CAT(0) spaces are another very important class of $W$-hyperbolic spaces. A $C A T(0)$ space is a geodesic space satisfying the CN inequality of Bruhat-Tits [8]: for all $x, y, z \in X$ and all $m \in X$ with $d(x, m)=d(y, m)=\frac{1}{2} d(x, y)$,

$$
d(z, m)^{2} \leq \frac{1}{2} d(z, x)^{2}+\frac{1}{2} d(z, y)^{2}-\frac{1}{4} d(x, y)^{2} .
$$

We refer to [4, p. 163] for a proof that the above definition is equivalent with the one using geodesic triangles. In the setting of $W$-hyperbolic spaces, we consider the following reformulation of the $\mathbf{C N}$ inequality: for all $x, y, z \in X$,

$$
\mathbf{C N}^{-}: d\left(z, W\left(x, y, \frac{1}{2}\right)\right)^{2} \leq \frac{1}{2} d(z, x)^{2}+\frac{1}{2} d(z, y)^{2}-\frac{1}{4} d(x, y)^{2} .
$$

We refer to [25, pp. 386-388] for the proof of the following result.

Proposition 2.8. Let $(X, d)$ be a metric space. The following statements are equivalent:

(i) $X$ is a $C A T(0)$ space.

(ii) There exists a (unique) convexity mapping $W$ such that $(X, d, W)$ is a $W$-hyperbolic space satisfying the $\mathbf{C N}^{-}$inequality (14).

Convention. Given a W-hyperbolic space $(X, d, W)$ and $x, y \in X, \lambda \in[0,1]$, we shall use from now on the notation $(1-\lambda) x \oplus \lambda y$ for $W(x, y, \lambda)$.

2.1. UCW-hyperbolic spaces. We define uniform convexity in the setting of $W$ hyperbolic spaces, following [18, p. 105]. Thus, a $W$-hyperbolic space $(X, d, W)$ is uniformly convex [29] if for any $r>0$ and any $\varepsilon \in(0,2]$ there exists $\delta \in(0,1]$ such that for all $a, x, y \in X$,

$$
\left.\begin{array}{l}
d(x, a) \leq r \\
d(y, a) \leq r \\
d(x, y) \geq \varepsilon r
\end{array}\right\} \quad \Rightarrow \quad d\left(\frac{1}{2} x \oplus \frac{1}{2} y, a\right) \leq(1-\delta) r .
$$

A mapping $\eta:(0, \infty) \times(0,2] \rightarrow(0,1]$ providing such a $\delta:=\eta(r, \varepsilon)$ for given $r>0$ and $\varepsilon \in(0,2]$ is called a modulus of uniform convexity. We call $\eta$ monotone if it decreases with $r$ (for a fixed $\varepsilon$ ).

Proposition 2.9. Any uniformly convex $W$-hyperbolic space is a Busemann space.

Proof. Apply [29, Proposition 5], Proposition 2.5(ii) and Proposition 2.6.

Following $[30$, we shall refer to uniformly convex $W$-hyperbolic spaces with a monotone modulus of uniform convexity as $U C W$-hyperbolic spaces. Furthermore, we shall also use the notation $(X, d, W, \eta)$ for a $U C W$-hyperbolic space having $\eta$ as a monotone modulus of uniform convexity.

As it was proved in 29, CAT(0) spaces are $U C W$-hyperbolic spaces with a modulus of uniform convexity $\eta(r, \varepsilon)=\frac{\varepsilon^{2}}{8}$ that does not depend on $r$ and is quadratic in $\varepsilon$. In particular, any $\mathrm{CAT}(0)$ space is also a Busemann space. 
The following lemma collects some useful properties of $U C W$-hyperbolic spaces. We refer to [29, 30, for the proofs.

Lemma 2.10. Let $(X, d, W, \eta)$ be a $U C W$-hyperbolic space. Assume that $r>0$, $\varepsilon \in(0,2]$ and $a, x, y \in X$ are such that

$$
d(x, a) \leq r, d(y, a) \leq r \text { and } d(x, y) \geq \varepsilon r .
$$

Let $\lambda \in[0,1]$ be arbitrary.

(i) $d((1-\lambda) x \oplus \lambda y, a) \leq(1-2 \lambda(1-\lambda) \eta(r, \varepsilon)) r$.

(ii) For any $0<\psi \leq \varepsilon$,

$$
d((1-\lambda) x \oplus \lambda y, a) \leq(1-2 \lambda(1-\lambda) \eta(r, \psi)) r .
$$

(iii) For any $s \geq r$,

$$
d((1-\lambda) x \oplus \lambda y, a) \leq(1-2 \lambda(1-\lambda) \eta(s, \varepsilon)) r .
$$

2.2. Asymptotic centers. One of the most useful tools in metric fixed point theory is the asymptotic center technique, introduced by Edelstein [11,12.

Let $(X, d)$ be a metric space, $\left(x_{n}\right)$ be a bounded sequence in $X$ and $C \subseteq X$ be a nonempty subset of $X$. We define the following functional:

$$
r\left(\cdot,\left(x_{n}\right)\right): X \rightarrow[0, \infty), \quad r\left(y,\left(x_{n}\right)\right)=\limsup _{n \rightarrow \infty} d\left(y, x_{n}\right) .
$$

The asymptotic radius of $\left(x_{n}\right)$ with respect to $C$ is given by

$$
r\left(C,\left(x_{n}\right)\right)=\inf \left\{r\left(y,\left(x_{n}\right)\right) \mid y \in C\right\} .
$$

A point $c \in C$ is said to be an asymptotic center of $\left(x_{n}\right)$ with respect to $C$ if

$$
r\left(c,\left(x_{n}\right)\right)=r\left(C,\left(x_{n}\right)\right)=\min \left\{r\left(y,\left(x_{n}\right)\right) \mid y \in C\right\} .
$$

We denote with $A\left(C,\left(x_{n}\right)\right)$ the set of asymptotic centers of $\left(x_{n}\right)$ with respect to $C$. When $C=X$, we call $c$ an asymptotic center of $\left(x_{n}\right)$ and we use the notation $A\left(\left(x_{n}\right)\right)$ for $A\left(X,\left(x_{n}\right)\right)$.

The following lemma will be very useful in the sequel.

Lemma 2.11 ([30]). Let $\left(x_{n}\right)$ be a bounded sequence in $X$ with $A\left(C,\left(x_{n}\right)\right)=\{c\}$ and let $\left(\alpha_{n}\right),\left(\beta_{n}\right)$ be real sequences such that $\alpha_{n} \geq 0$ for all $n \in \mathbb{N}$, $\lim \sup \alpha_{n} \leq 1$ and $\lim \sup \beta_{n} \leq 0$.

Assume that $y \in C$ is such that there exist $p, N \in \mathbb{N}$ satisfying

$$
\forall n \geq N\left(d\left(y, x_{n+p}\right) \leq \alpha_{n} d\left(c, x_{n}\right)+\beta_{n}\right) .
$$

Then $y=c$.

A classical result is the fact that in uniformly convex Banach spaces, bounded sequences have unique asymptotic centers with respect to closed convex subsets. For the Hilbert ball, this was proved in [18, Proposition 21.1]. The following result shows that the same is true for complete $U C W$-hyperbolic spaces.

Proposition 2.12 ([30]). Let $(X, d, W)$ be a complete $U C W$-hyperbolic space. Every bounded sequence $\left(x_{n}\right)$ in $X$ has a unique asymptotic center with respect to any nonempty closed convex subset $C$ of $X$. 
2.3. Convex functions. Let $(X, d)$ be a geodesic space and $F: X \rightarrow(-\infty, \infty]$. The mapping $F$ is said to be convex if, for any geodesic $\gamma$ in $X$, the function $F \circ \gamma$ is convex. Let us recall that the effective domain of $F$ is the set $\operatorname{dom} F:=\{x \in X \mid$ $F(x)<\infty\}$ and that $F$ is proper if $\operatorname{dom} F$ is nonempty.

For the rest of this section $F$ is a proper convex function.

If $x \in \operatorname{dom} F$ and $\gamma:[0, c] \rightarrow X$ is a geodesic starting at $x$, the directional derivative $D_{\gamma} F(x)$ of $F$ at $x$ in the direction $\gamma$ is defined by

$$
D_{\gamma} F(x):=\lim _{t \rightarrow 0^{+}} \frac{F(\gamma(t))-F(x)}{t} .
$$

As $F$ is convex, the above limit (possibly infinite) always exists. Indeed, one can easily see that $D_{\gamma} F(x)=\inf _{t>0} \frac{F(\gamma(t))-F(x)}{t}$.

Proposition 2.13. Let $\bar{x} \in \operatorname{dom} F$. The following statements are equivalent:

(i) $\bar{x}$ is a local minimum of $F$.

(ii) $\bar{x}$ is a global minimum of $F$.

(iii) $D_{\gamma} F(\bar{x}) \geq 0$ for any geodesic $\gamma:[0, c] \rightarrow X$ starting at $\bar{x}$.

Proof. $(i) \Rightarrow($ ii $)$ Let $\varepsilon>0$ be such that $F(\bar{x}) \leq F(x)$ for all $x \in B(\bar{x}, \varepsilon)$. Let $z \neq \bar{x}$ be arbitrary and $\gamma:[0, d(\bar{x}, z)] \rightarrow X$ be a geodesic in $X$ that joins $\bar{x}$ and $z$. For all $t<\min \{\varepsilon, d(\bar{x}, z)\}$ we have that $d(\gamma(t), \bar{x})=t<\varepsilon$, so that

$$
\begin{aligned}
F(\bar{x}) & \leq F(\gamma(t))=(F \circ \gamma)\left(\left(1-\frac{t}{d(\bar{x}, z)}\right) 0+\frac{t}{d(\bar{x}, z)} d(\bar{x}, z)\right) \\
& \leq\left(1-\frac{t}{d(\bar{x}, z)}\right) F(\bar{x})+\frac{t}{d(\bar{x}, z)} F(z) .
\end{aligned}
$$

Hence $F(\bar{x}) \leq F(z)$.

$($ ii $) \Rightarrow(i)$ and $(i i) \Rightarrow(i i i)$ are immediate.

(iii) $\Rightarrow($ ii $)$ Let $z \neq \bar{x}$ be arbitrary. We shall prove that $F(z) \geq F(\bar{x})$. If $F(z)=\infty$, the conclusion is obvious, so we can assume that $z \in \operatorname{dom} F$. Let $\gamma:[0, d(\bar{x}, z)] \rightarrow X$ be a geodesic that joins $\bar{x}$ and $z$. As $F$ is convex, one gets that for all $t \in[0, d(\bar{x}, z)]$,

$$
F(\gamma(t)) \leq\left(1-\frac{t}{d(\bar{x}, z)}\right) F(\bar{x})+\frac{t}{d(\bar{x}, z)} F(z)<\infty .
$$

Thus, $F \circ \gamma:[0, d(\bar{x}, z)] \rightarrow \mathbb{R}$ is a convex real function satisfying $(F \circ \gamma)^{\prime}(0)=$ $D_{\gamma} F(\bar{x}) \geq 0$. One gets that $F(\bar{x})=(F \circ \gamma)(0) \leq(F \circ \gamma)(d(\bar{x}, z))=F(z)$.

\section{FiRMLY NONEXPANSIVE MAPPINGS}

Firmly nonexpansive mappings were introduced by Bruck 6 in the context of Banach spaces and by Browder [5], under the name firmly contractive, in the setting of Hilbert spaces. We refer to [18, Section 24] for a study of this class of mappings in the Hilbert ball.

Bruck's definition can be extended to $W$-hyperbolic spaces. Let $(X, d, W)$ be a $W$-hyperbolic space, $C \subseteq X$ and $T: C \rightarrow X$. Given $\lambda \in(0,1)$, we say that $T$ is $\lambda$-firmly nonexpansive if for all $x, y \in C$,

$$
d(T x, T y) \leq d((1-\lambda) x \oplus \lambda T x,(1-\lambda) y \oplus \lambda T y) \text { for all } x, y \in C .
$$

If (16) holds for all $\lambda \in(0,1)$, then $T$ is said to be firmly nonexpansive. 
Applying (W4) one gets that any $\lambda$-firmly nonexpansive mapping is nonexpansive, i.e. it satisfies $d(T x, T y) \leq d(x, y)$ for all $x, y \in C$.

The first example of a firmly nonexpansive mapping is the metric projection in a CAT(0) space. Let us recall that a subset $C$ of a metric space $(X, d)$ is called a Chebyshev set if to each point $x \in X$ there corresponds a unique point $z \in C$ such that $d(x, z)=d(x, C)$, where $d(x, C)=\inf \{d(x, y) \mid y \in C\}$. If $C$ is a Chebyshev set, the metric projection $P_{C}: X \rightarrow C$ can be defined by assigning $z$ to $x$.

By [4, Proposition II.2.4], any closed convex subset $C$ of a $\mathrm{CAT}(0)$ space is a Chebyshev set, the metric projection $P_{C}$ is nonexpansive and $P_{C}\left((1-\lambda) x \oplus \lambda P_{C} x\right)=$ $P_{C}(x)$ for all $x \in X$ and all $\lambda \in(0,1)$. It is well known that in the setting of Hilbert spaces the metric projection is firmly nonexpansive. We remark that for the Hilbert ball this was proved in [18, p. 111]. The following result shows that the same holds in general $\mathrm{CAT}(0)$ spaces.

Proposition 3.1. Let $C$ be a nonempty closed convex subset of a $C A T(0)$ space $(X, d)$. The metric projection $P_{C}$ onto $C$ is a firmly nonexpansive mapping.

Proof. Let $x, y \in X$ and $\lambda \in(0,1)$. One gets that

$$
\begin{aligned}
d\left(P_{C} x, P_{C} y\right) & =d\left(P_{C}\left((1-\lambda) x \oplus \lambda P_{C} x\right), P_{C}\left((1-\lambda) y \oplus \lambda P_{C} y\right)\right) \\
& \leq d\left((1-\lambda) x \oplus \lambda P_{C} x,(1-\lambda) y \oplus \lambda P_{C} y\right) .
\end{aligned}
$$

Bruck [6] showed for Banach spaces that one can associate to any nonexpansive mapping a family of firmly nonexpansive mappings having the same fixed points. Goebel and Reich [18] obtained the same result for the Hilbert ball. We show in the sequel that Bruck's construction can also be adapted to Busemann spaces.

Let $C$ be a nonempty closed convex subset of a complete Busemann space $X$ and let $T: C \rightarrow C$ be nonexpansive. For $t \in(0,1)$ and $x \in C$ define

$$
T_{t}^{x}: C \rightarrow C, \quad T_{t}^{x}(y)=(1-t) x \oplus t T(y) .
$$

Using (W4), one can easily see that $T_{t}^{x}$ is a contraction, so it has a unique fixed point $z_{t}^{x} \in C$, by Banach's Contraction Mapping Principle. Let

$$
U_{t}: C \rightarrow C, \quad U_{t}(x)=z_{t}^{x} .
$$

Then $U_{t}(x)=(1-t) x \oplus t T\left(U_{t}(x)\right)$ for all $x \in C$.

Proposition 3.2. $U_{t}$ is a firmly nonexpansive mapping having the same set of fixed points as $T$.

Proof. Let $\lambda \in(0,1)$ and $x, y \in C$. Denote $u:=(1-\lambda) x \oplus \lambda U_{t}(x)$ and $v:=$ $(1-\lambda) y \oplus \lambda U_{t}(y)$. We can apply Lemma 2.4(iii) twice to get that

$U_{t}(x)=(1-\mu) u \oplus \mu T\left(U_{t}(x)\right), \quad U_{t}(y)=(1-\mu) v \oplus \mu T\left(U_{t}(y)\right)$, where $\mu=\frac{(1-\lambda) t}{1-\lambda t}$.

It follows that

$$
\begin{aligned}
d\left(U_{t}(x), U_{t}(y)\right) & =d\left((1-\mu) u \oplus \mu T\left(U_{t}(x)\right),(1-\mu) v \oplus \mu T\left(U_{t}(y)\right)\right) \\
& \leq(1-\mu) d(u, v)+\mu d\left(T\left(U_{t}(x)\right), T\left(U_{t}(y)\right)\right) \\
& \leq(1-\mu) d(u, v)+\mu d\left(U_{t}(x), U_{t}(y)\right) .
\end{aligned}
$$

Thus, $d\left(U_{t}(x), U_{t}(y)\right) \leq d(u, v)$, so $U_{t}$ is $\lambda$-firmly nonexpansive.

The fact that $U_{t}$ and $T$ have the same set of fixed points is immediate. 
A third example of a firmly nonexpansive mapping is the resolvent of a proper, convex and lower semicontinuous mapping in a CAT(0) space.

Let $(X, d)$ be a $\operatorname{CAT}(0)$ space, $F: X \rightarrow(-\infty, \infty]$ and $\mu>0$. Following Jost [20], the Moreau-Yosida approximation $F^{\mu}$ of $F$ is defined by

$$
F^{\mu}(x):=\inf _{y \in X}\left\{\mu F(y)+d(x, y)^{2}\right\} .
$$

We refer to [1,45] for applications of the Moreau-Yosida approximation in $\operatorname{CAT}(0)$ spaces.

Jost proved [20, Lemma 2] that if $F: X \rightarrow(-\infty, \infty]$ is proper, convex and lower semicontinuous, then for every $x \in X$ and $\mu>0$ there exists a unique $y_{\mu} \in X$ such that

$$
F^{\mu}(x)=\mu F\left(y_{\mu}\right)+d\left(x, y_{\mu}\right)^{2} .
$$

We denote this $y_{\mu}$ with $J_{\mu}(x)$ and call $J_{\mu}$ the resolvent of $F$ of order $\mu$.

In the same paper, Jost shows that for all $\mu>0$ the resolvent $J_{\mu}$ is nonexpansive [20. Lemma 4] and, furthermore, that for all $\lambda \in[0,1]$,

$$
J_{(1-\lambda) \mu}\left((1-\lambda) x \oplus \lambda J_{\mu}(x)\right)=J_{\mu}(x) \quad(\text { see [20, Corollary 1]). }
$$

Proposition 3.3. Let $F: X \rightarrow(-\infty, \infty]$ be proper, convex and lower semicontinuous. Then for every $\mu>0$, its resolvent $J_{\mu}$ is a firmly nonexpansive mapping.

Proof. Let $x, y \in X$ and $\lambda \in(0,1)$. Then

$$
\begin{aligned}
d\left(J_{\mu}(x), J_{\mu}(y)\right) & =d\left(J_{(1-\lambda) \mu}\left((1-\lambda) x \oplus \lambda J_{\mu}(x)\right), J_{(1-\lambda) \mu}\left((1-\lambda) y \oplus \lambda J_{\mu}(y)\right)\right) \\
& \leq d\left((1-\lambda) x \oplus \lambda J_{\mu}(x),(1-\lambda) y \oplus \lambda J_{\mu}(y)\right) .
\end{aligned}
$$

Another example of a firmly nonexpansive mapping, given by Kopecká and Reich [27. Lemma 2.2], is the resolvent of a coaccretive operator in the Hilbert ball.

\section{A FIXED POINT THEOREM}

Given a subset $C$ of a metric space $(X, d)$, a nonexpansive mapping $T: C \rightarrow C$ and $x \in C$, the orbit $\mathcal{O}(x)$ of $x$ under $T$ is defined by $\mathcal{O}(x)=\left\{T^{n} x \mid n=0,1,2, \ldots\right\}$. As an immediate consequence of the nonexpansiveness of $T$, if $\mathcal{O}(x)$ is bounded for some $x \in C$, then all other orbits $\mathcal{O}(y), y \in C$, are bounded. If this is the case, we say that $T$ has bounded orbits. Obviously, if $T$ has fixed points, then $T$ has bounded orbits.

In this section we prove the following fixed point theorem.

Theorem 4.1. Let $(X, d, W)$ be a complete $U C W$-hyperbolic space, $C=\bigcup_{k=1}^{p} C_{k}$ be a union of nonempty closed convex subsets $C_{k}$ of $X$, and $T: C \rightarrow C$ be $\lambda$-firmly nonexpansive for some $\lambda \in(0,1)$. The following two statements are equivalent:

(i) $T$ has bounded orbits.

(ii) $T$ has fixed points.

Let us remark that fixed points are not guaranteed if $T$ is merely nonexpansive, as the following trivial example shows. Let $x \neq y \in X$, take $C_{1}=\{x\}, C_{2}=$ $\{y\}, C=C_{1} \cup C_{2}$ and $T: C \rightarrow C, T(x)=y, T(y)=x$. Then $T$ is fixed point free 
and nonexpansive. If $T$ were $\lambda$-firmly nonexpansive for some $\lambda \in(0,1)$, we would get

$$
\begin{aligned}
0<d(x, y) & =d(T x, T y) \leq d((1-\lambda) x \oplus \lambda T x,(1-\lambda) y \oplus \lambda T y) \\
& =d((1-\lambda) x \oplus \lambda y, \lambda x \oplus(1-\lambda) y)=|2 \lambda-1| d(x, y) \quad \text { by (W2) } \\
& <d(x, y),
\end{aligned}
$$

that is a contradiction.

As an immediate consequence, we get a strengthening of Smarzewski's fixed point theorem for uniformly convex Banach spaces [43, obtained by weakening the hypothesis of $C_{k}$ being bounded for all $k=1, \ldots, p$ to $T$ having bounded orbits.

Corollary 4.2. Let $X$ be a uniformly convex Banach space, $C=\bigcup_{k=1}^{p} C_{k}$ be a union of nonempty closed convex subsets $C_{k}$ of $X$, and $T: C \rightarrow C$ be $\lambda$-firmly nonexpansive for some $\lambda \in(0,1)$.

Then $T$ has fixed points if and only if $T$ has bounded orbits.

Theorem 4.1 follows from the following Propositions 4.5 and 4.3

Proposition 4.3. Let $X$ be a Busemann space, $C \subseteq X$ be nonempty and $T: C \rightarrow$ $C$ be $\lambda$-firmly nonexpansive for some $\lambda \in(0,1)$. Then any periodic point of $T$ is a fixed point of $T$.

Proof. Let $x$ be a periodic point of $T$ and $m \geq 0$ be minimal with the property that $T^{m+1} x=x$. If $m=0$, then $x$ is a fixed point of $T$, hence we can assume that $m \geq 1$. Since $T$ is nonexpansive, we have

$$
\begin{aligned}
d\left(x, T^{m} x\right) & =d\left(T^{m+1} x, T^{m} x\right) \leq d\left(T^{m} x, T^{m-1} x\right) \leq \ldots \leq d(T x, x) \\
& =d\left(T x, T^{m+1} x\right) \leq d\left(x, T^{m} x\right)
\end{aligned}
$$

hence we must have equality everywhere, that is,

$$
d(T x, x)=d\left(T^{2} x, T x\right)=\ldots=d\left(T^{m} x, T^{m-1} x\right)=d\left(x, T^{m} x\right):=\gamma>0,
$$

since $T^{m} x \neq x$, by the hypothesis on $m$. Now applying the fact that $T$ is $\lambda$-firmly nonexpansive, we get for all $k=1, \ldots, m$

$$
\begin{aligned}
\gamma & =d\left(T^{k+1} x, T^{k} x\right) \leq d\left((1-\lambda) T^{k} x \oplus \lambda T^{k+1} x,(1-\lambda) T^{k-1} x \oplus \lambda T^{k} x\right) \\
& \leq d\left((1-\lambda) T^{k} x \oplus \lambda T^{k+1} x, T^{k} x\right)+d\left(T^{k} x,(1-\lambda) T^{k-1} x \oplus \lambda T^{k} x\right) \\
& =\lambda d\left(T^{k} x, T^{k+1} x\right)+(1-\lambda) d\left(T^{k-1} x, T^{k} x\right)=\lambda \gamma+(1-\lambda) \gamma=\gamma
\end{aligned}
$$

Hence, we must have

$$
\gamma=d\left(\alpha_{k}, \beta_{k}\right)=d\left(\alpha_{k}, T^{k} x\right)+d\left(T^{k} x, \beta_{k}\right),
$$

where

$$
\begin{aligned}
\alpha_{k} & :=(1-\lambda) T^{k} x \oplus \lambda T^{k+1} x, \\
\beta_{k} & :=(1-\lambda) T^{k-1} x \oplus \lambda T^{k} x=\lambda T^{k} x \oplus(1-\lambda) T^{k-1} x .
\end{aligned}
$$

We have the following cases:

(i) $m=1$, hence $k=1$. Then $T^{m-1} x=x$ and

$$
\alpha_{1}=(1-\lambda) T x \oplus \lambda x, \quad \beta_{1}=\lambda T x \oplus(1-\lambda) x .
$$


It follows by (W2) that

$$
\gamma=d\left(\alpha_{1}, \beta_{1}\right)=|\lambda-(1-\lambda)| d(x, T x)=|2 \lambda-1| \gamma,
$$

hence $|2 \lambda-1|=1$, which is impossible, since $\lambda \in(0,1)$.

(ii) $m \geq 2$, hence $m-1 \geq 1$. Since $T^{k} x$ lies between $\beta_{k}$ and $\alpha_{k}$ and, furthermore, $\alpha_{k}$ lies between $T^{k} x$ and $T^{k+1} x$, we can apply Lemma 2.7 twice to get first that $T^{k} x$ lies between $\beta_{k}$ and $T^{k+1} x$ and second, since $\beta_{k}$ lies between $T^{k-1} x$ and $T^{k} x$, that $T^{k} x$ lies between $T^{k-1} x$ and $T^{k+1} x$ for all $k=1, \ldots, m$. hence

Now apply Lemma2.2 to conclude that $T^{m-1} x$ lies between $x$ and $T^{m} x$, $\gamma=d\left(x, T^{m} x\right)=d\left(T^{m-1} x, T^{m} x\right)+d\left(T^{m-1} x, x\right)=\gamma+d\left(T^{m-1} x, x\right)>\gamma$, since $T^{m-1} x \neq x$. We have a contradiction.

We remark that Proposition 4.3 holds for strictly convex Banach spaces too, as they are Busemann spaces.

Lemma 4.4. Let $(X, d)$ be a metric space, $C=\bigcup_{k=1}^{p} C_{k}$ be a union of nonempty subsets $C_{k}$ of $X$, and $T: C \rightarrow C$ be nonexpansive. Assume that $T$ has bounded orbits and that for some $z \in C$, the orbit $\left(T^{n} z\right)$ of $T$ has a unique asymptotic center $x_{k}$ with respect to every $C_{k}, k=1, \ldots, p$.

Then one of $x_{k}, k=1, \ldots, p$, is a periodic point of $T$.

Proof. Since $T$ is nonexpansive, we have that

$$
\begin{aligned}
d\left(T x_{k}, T^{n+1} z\right) & \leq d\left(x_{k}, T^{n} z\right) \text { for all } n \geq 0, k=1, \ldots, p, \text { hence } \\
r\left(T x_{k},\left(T^{n} z\right)\right) & \leq r\left(x_{k},\left(T^{n} z\right)\right) \text { for all } k=1, \ldots, p .
\end{aligned}
$$

If there exists $k_{0} \in\{1, \ldots, p\}$ such that $T x_{k_{0}} \in C_{k_{0}}$, then applying Lemma 2.11 with $y=T x_{k_{0}}, p=1, \alpha_{n}=1, \beta_{n}=0$ and $x_{n}=T^{n} z$, we have that $T x_{k_{0}}=x_{k_{0}}$, that is, $x_{k_{0}}$ is a fixed point of $T$. In particular, $x_{k_{0}}$ is a periodic point of $T$.

Otherwise, assume that $T x_{k} \notin C_{k}$ for all $1 \leq k \leq p$. It is easy to see that there exist integers $\left\{n_{1}, n_{2}, \ldots, n_{m}\right\} \subseteq\{1,2, \ldots, p\}$, with $m \geq 2$, such that $T x_{n_{k}} \in C_{n_{k+1}}$ for all $k=1, \ldots, m-1$ and $T x_{n_{m}} \in C_{n_{1}}$.

Applying (24) repeatedly, and the fact that $x_{n_{k}}$ is the unique asymptotic center of $\left(T^{n} z\right)$ with respect to $C_{n_{k}}$, we get that

$$
\begin{aligned}
r\left(x_{n_{1}},\left(T^{n} z\right)\right) & \leq r\left(T x_{n_{m}},\left(T^{n} z\right)\right) \leq r\left(x_{n_{m}},\left(T^{n} z\right)\right) \leq \ldots \leq r\left(T x_{n_{1}},\left(T^{n} z\right)\right) \\
& \leq r\left(x_{n_{1}},\left(T^{n} z\right)\right) .
\end{aligned}
$$

Thus, we must have equality everywhere. We obtain $r\left(x_{n_{1}},\left(T^{n} z\right)\right)=r\left(T x_{n_{m}},\left(T^{n} z\right)\right)$ and $r\left(T x_{n_{k}},\left(T^{n} z\right)\right)=r\left(x_{n_{k+1}},\left(T^{n} z\right)\right)$ for all $k=1, \ldots, m-1$. By the uniqueness of the asymptotic centers, we get that

$$
x_{n_{1}}=T x_{n_{m}} \text { and } T x_{n_{k}}=x_{n_{k+1}} \text { for all } k=1, \ldots, m-1 .
$$

It follows that $T^{m} x_{n_{1}}=x_{n_{1}}$, hence $x_{n_{1}}$ is a periodic point of $T$.

Proposition 4.5. Let $(X, d, W)$ be a complete $U C W$-hyperbolic space, $C=\bigcup_{k=1}^{p} C_{k}$ be a union of nonempty closed convex subsets $C_{k}$ of $X$, and $T: C \rightarrow \stackrel{k=1}{C}$ be $a$ nonexpansive mapping having bounded orbits. 
Then T has periodic points.

Proof. By Proposition 2.12, for all $z \in C$ and for all $k=1, \ldots, p$, the orbit $\left(T^{n} z\right)$ has a unique asymptotic center $x_{k}$ with respect to $C_{k}$. Apply Lemma 4.4 to get that one of the asymptotic centers $x_{k}, k=1, \ldots, p$, is a periodic point of $T$.

\section{Asymptotic Behaviour of Picard iterations}

The second main result of the paper is a theorem on the asymptotic behaviour of Picard iterations of $\lambda$-firmly nonexpansive mappings, which generalizes results obtained by Reich and Shafrir [40] for firmly nonexpansive mappings in Banach spaces and the Hilbert ball.

Theorem 5.1. Let $C$ be a subset of a $W$-hyperbolic space $X$ and $T: C \rightarrow C$ be $a$ $\lambda$-firmly nonexpansive mapping with $\lambda \in(0,1)$. Then for all $x \in X$ and $k \in \mathbb{Z}_{+}$,

$$
\lim _{n \rightarrow \infty} d\left(T^{n+1} x, T^{n} x\right)=\frac{1}{k} \lim _{n \rightarrow \infty} d\left(T^{n+k} x, T^{n} x\right)=\lim _{n \rightarrow \infty} \frac{d\left(T^{n} x, x\right)}{n}=r_{C}(T),
$$

where $r_{C}(T):=\inf \{d(x, T x) \mid x \in C\}$ is the minimal displacement of $T$.

The mapping $T$ is said to be asymptotically regular at $x \in C$ if $\lim _{n \rightarrow \infty} d\left(T^{n} x, T^{n+1} x\right)$ $=0$. If this is true for all $x \in C$, we say that $T$ is asymptotically regular.

Before proving Theorem 5.1, we give the following immediate consequences.

Corollary 5.2. The following statements are equivalent:

(i) $T$ is asymptotically regular at some $x \in C$.

(ii) $r_{C}(T)=0$.

(iii) $T$ is asymptotically regular.

Corollary 5.3. If $T$ has bounded orbits, then $T$ is asymptotically regular.

Remark 5.4. As Adriana Nicolae pointed out to us in a private communication, one can easily see that Proposition 4.3 is an immediate consequence of the above corollary. However, our proof of this proposition also holds (with small adaptations) in more general spaces like geodesic spaces with the betweenness property (see [37]), for which it is not known whether Corollary 5.3 is true.

5.1. Proof of Theorem 5.1. In the sequel, $X$ is a $W$-hyperbolic space, $C \subseteq X$ and $T: C \rightarrow C$.

Lemma 5.5. Assume that $T$ is nonexpansive and $x \in C$.

(i) For all $k \geq 1, R_{k}:=\lim _{n \rightarrow \infty} d\left(T^{n+k} x, T^{n} x\right)$ exists and $R_{k} \leq k R_{1}$.

(ii) $L:=\lim _{n \rightarrow \infty} \frac{d\left(T^{n} x, x\right)}{n}$ exists and equals $\inf _{n \geq 1} \frac{d\left(T^{n} x, x\right)}{n}$. Moreover, $L$ is independent of $x$.

(iii) $L \leq r_{C}(T) \leq R_{1}$.

Proof. (i) Since the sequence $\left(d\left(T^{n+k} x, T^{n} x\right)\right)_{n}$ is nonincreasing, obviously $R_{k}$ exists. Remark that

$$
d\left(T^{n+k} x, T^{n} x\right) \leq \sum_{i=0}^{k-1} d\left(T^{n+i} x, T^{n+i+1} x\right) \leq k d\left(T^{n+1} x, T^{n} x\right)
$$

and let $n \rightarrow \infty$ to conclude that $R_{k} \leq k R_{1}$. 
(ii) One has that for all $m, n \geq 1$,

$$
d\left(T^{m+n} x, x\right) \leq d\left(T^{m+n} x, T^{n} x\right)+d\left(T^{n} x, x\right) \leq d\left(T^{m} x, x\right)+d\left(T^{n} x, x\right)
$$

hence the sequence $\left(d\left(T^{n} x, x\right)\right)$ is subadditive. Now apply Fekete's subadittive lemma [14] to get that $L=\inf _{n \geq 1} \frac{d\left(T^{n} x, x\right)}{n}$. The independence of $x$ follows from the fact that for all $x, y \in C$,

$$
d\left(T^{n} x, x\right)-d\left(T^{n} y, y\right) \leq d\left(T^{n} x, T^{n} y\right)+d(x, y) \leq 2 d(x, y) .
$$

(iii) Obviously, $R_{1}=\inf _{n \geq 1} d\left(T^{n} x, T^{n+1} x\right) \geq r_{C}(T)$. Given $\varepsilon>0$, there is a point $y \in C$ such that $r_{C}(T) \leq d(y, T y)<r_{C}(T)+\varepsilon$. It follows that

$$
\begin{aligned}
L & =\lim _{n \rightarrow \infty} \frac{d\left(T^{n} x, x\right)}{n}=\lim _{n \rightarrow \infty} \frac{d\left(T^{n} y, y\right)}{n}=\inf _{n \geq 1} \frac{d\left(T^{n} y, y\right)}{n} \leq d(T y, y) \\
& <r_{C}(T)+\varepsilon .
\end{aligned}
$$

As $\varepsilon>0$ was arbitrary, we get that $L \leq r_{C}(T)$.

Lemma 5.6. Let $T$ be $\lambda$-firmly nonexpansive for some $\lambda \in(0,1)$. Then for all $x, y \in C$,

$$
d(T x, T y) \leq \frac{1-\lambda}{1+\lambda} d(x, y)+\frac{\lambda}{1+\lambda}(d(T x, y)+d(x, T y)) .
$$

Proof. Apply (W1) additional times to get that

$$
\begin{aligned}
d(T x, T y) & \leq d((1-\lambda) x \oplus \lambda T x,(1-\lambda) y \oplus \lambda T y) \\
& \leq(1-\lambda) d((1-\lambda) x \oplus \lambda T x, y)+\lambda d((1-\lambda) x \oplus \lambda T x, T y) \\
& \leq(1-\lambda)^{2} d(x, y)+\lambda(1-\lambda)(d(T x, y)+d(x, T y))+\lambda^{2} d(T x, T y) .
\end{aligned}
$$

Proof of Theorem 5.1. We prove that $R_{k}=k R_{1}$ for all $k \geq 1$ by induction on $k$. Assume that $R_{j}=j R_{1}$ for all $j=1, \ldots, k$ and let $\varepsilon>0$. Since $\left(d\left(T^{n+j} x, T^{n} x\right)\right)$ is nonincreasing, we get $N_{\varepsilon} \geq 1$ such that for any $j=1, \ldots, k$ and for all $n \geq N_{\varepsilon}$,

$$
R_{1} \leq \frac{1}{j} d\left(T^{n+j} x, T^{n} x\right) \leq R_{1}+\varepsilon
$$

Let $n \geq N_{\varepsilon}$. By (26), we get that

$$
\begin{aligned}
d\left(T^{n+1} x, T^{n+k+1} x\right) \leq & \frac{1-\lambda}{1+\lambda} d\left(T^{n} x, T^{n+k} x\right) \\
& +\frac{\lambda}{1+\lambda}\left(d\left(T^{n+1} x, T^{n+k} x\right)+d\left(T^{n} x, T^{n+k+1} x\right)\right) ;
\end{aligned}
$$

hence

$$
\begin{aligned}
d\left(T^{n} x, T^{n+k+1} x\right) \geq & \frac{1+\lambda}{\lambda} d\left(T^{n+1} x, T^{n+k+1} x\right)-\frac{1-\lambda}{\lambda} d\left(T^{n+k} x, T^{n} x\right) \\
& -d\left(T^{n+1} x, T^{n+k} x\right) \\
\geq & \frac{1+\lambda}{\lambda} k R_{1}-\frac{1-\lambda}{\lambda} k\left(R_{1}+\varepsilon\right)-(k-1)\left(R_{1}+\varepsilon\right) \\
= & (k+1) R_{1}+\left(1-\frac{k}{\lambda}\right) \varepsilon .
\end{aligned}
$$


By letting $n \rightarrow \infty$, it follows that $R_{k+1} \geq(k+1) R_{1}$, as $\varepsilon>0$ is arbitrary. Now apply Lemma 5.5(1) to conclude that $R_{k+1}=(k+1) R_{1}$.

Since $d\left(T^{n+k} x, T^{n} x\right) \leq d\left(T^{k} x, x\right)$ for all $k, n \geq 1$, let $n \rightarrow \infty$ to get that $R_{1} \leq$ $\frac{d\left(T^{k} x, x\right)}{k}$ for all $k \geq 1$ and, as a consequence, $R_{1} \leq L$. Apply now Lemma 5.5.(iii) to conclude that $L=R_{1}=r_{C}(T)$.

\section{6. $\Delta$-CONVERGENCE OF PiCARD ITERATES}

In 1976, Lim 32 introduced a concept of convergence in the general setting of metric spaces, which is known as $\Delta$-convergence. Kuczumow 28, introduced an identical notion of convergence in Banach spaces, which he called almost convergence. As shown in [22], $\Delta$-convergence could be regarded, at least for $\operatorname{CAT}(0)$ spaces, as an analogue to the usual weak convergence in Banach spaces. Jost [19] introduced a notion of weak convergence in CAT(0) spaces, which was rediscovered by Espínola and Fernández-León [13, who also proved that it is equivalent to $\Delta$-convergence. We refer to [4] for other notions of weak convergence in geodesic spaces.

Let $\left(x_{n}\right)$ be a bounded sequence of a metric space $(X, d)$. We say that $\left(x_{n}\right)$ $\Delta$-converges to $x$ if $x$ is the unique asymptotic center of $\left(u_{n}\right)$ for every subsequence $\left(u_{n}\right)$ of $\left(x_{n}\right)$. In this case, we write $x_{n} \stackrel{\Delta}{\longrightarrow} x$ or $\Delta-\lim _{n \rightarrow \infty} x_{n}=x$ and we call $x$ the $\Delta$-limit of $\left(x_{n}\right)$.

Let $(X, d)$ be a metric space and $F \subseteq X$ be a nonempty subset. A sequence $\left(x_{n}\right)$ in $X$ is said to be Fejér monotone with respect to $F$ if

$$
d\left(p, x_{n+1}\right) \leq d\left(p, x_{n}\right) \quad \text { for all } p \in F \text { and } n \geq 0 .
$$

Thus each point in the sequence is not further from any point in $F$ than its predecessor. Obviously, any Fejér monotone sequence $\left(x_{n}\right)$ is bounded, and moreover $\left(d\left(x_{n}, p\right)\right)$ converges for every $p \in F$.

The following lemma is very easy to prove.

Lemma 6.1. Let $(X, d)$ be a metric space, $F \subseteq X$ be a nonempty subset and $\left(x_{n}\right)$ be Fejér monotone with respect to $F$. Then

(i) For all $p \in F,\left(d\left(p, x_{n}\right)\right)$ converges and $r\left(p,\left(x_{n}\right)\right)=\lim _{n \rightarrow \infty} d\left(p, x_{n}\right)$.

(ii) Every subsequence $\left(u_{n}\right)$ of $\left(x_{n}\right)$ is Fejér monotone with respect to $F$, and for all $p \in F, r\left(p,\left(u_{n}\right)\right)=r\left(p,\left(x_{n}\right)\right)$. Hence, $r\left(F,\left(u_{n}\right)\right)=r\left(F,\left(x_{n}\right)\right)$ and $A\left(F,\left(u_{n}\right)\right)=A\left(F,\left(x_{n}\right)\right)$.

(iii) If $A\left(F,\left(x_{n}\right)\right)=\{x\}$ and $A\left(\left(u_{n}\right)\right) \subseteq F$ for every subsequence $\left(u_{n}\right)$ of $\left(x_{n}\right)$, then $\left(x_{n}\right) \Delta$-converges to $x \in F$.

Furthermore, one has the following result, whose proof is very similar to the one in strictly convex Banach spaces. For the sake of completeness, we give it here.

Lemma 6.2. Let $C$ be a nonempty closed convex subset of a uniquely geodesic space $(X, d)$ and $T: C \rightarrow C$ be nonexpansive. Then the set Fix $(T)$ of fixed points of $T$ is closed and convex.

Proof. The fact that Fix $(T)$ is closed is immediate from the continuity of $T$. We shall prove its convexity. Let $x, y \in F i x(T)$ be distinct and $z \in[x, y]$. Then

$d(x, y) \leq d(x, T z)+d(T z, y)=d(T x, T z)+d(T z, T y) \leq d(x, z)+d(z, y)=d(x, y)$. 
Thus, $d(x, T z)+d(T z, y)=d(x, y)$, so that $T z \in[x, y]$. We apply now Lemma 2.3 (iii) to get the following cases:

$$
\begin{aligned}
\text { (i) } \quad d(x, z)+d(z, T z) & =d(x, T z)=d(T x, T z) \leq d(x, z), \\
(i i) \quad d(y, z)+d(z, T z) & =d(y, T z)=d(T y, T z) \leq d(y, z) .
\end{aligned}
$$

In both cases, it follows that $T z=z$.

Proposition 6.3. Let $(X, d, W)$ be a complete $U C W$-hyperbolic space, $C \subseteq X$ be nonempty closed convex and $T: C \rightarrow C$ be a nonexpansive mapping with $F i x(T) \neq$ $\emptyset$. If $T$ is asymptotically regular at $x \in C$, then the Picard iterate $\left(T^{n} x\right) \Delta$-converges to a fixed point of $T$.

Proof. By Lemma 6.2 the nonempty set $F:=F i x(T)$ is closed and convex. Furthermore, one can see easily that $\left(T^{n} x\right)$ is Fejér monotone with respect to $F$ and, by Proposition 2.12, $\left(T^{n} x\right)$ has a unique asymptotic center with respect to $F$. Let $\left(u_{n}\right)$ be a subsequence of $\left(T^{n} x\right)$ and $u$ be its unique asymptotic center. Then

$$
d\left(T u, u_{n}\right) \leq d\left(T u, T u_{n}\right)+d\left(T u_{n}, u_{n}\right) \leq d\left(u, u_{n}\right)+d\left(u_{n}, T u_{n}\right),
$$

so we can use Lemma 2.11 to obtain that $T u=u$, i.e. $u \in F$. Apply Lemma 6.1(iii) to get the conclusion.

By [30, Theorem 3.5] one can replace in the above theorem the assumption that $T$ has fixed points with the equivalent one that $T$ has bounded orbits.

We get the following $\Delta$-convergence result for the Picard iteration of a firmly nonexpansive mapping.

Theorem 6.4. Let $(X, d, W)$ be a complete $U C W$-hyperbolic space, $C \subseteq X$ be nonempty closed convex and $T: C \rightarrow C$ be a $\lambda$-firmly nonexpansive mapping for some $\lambda \in(0,1)$. Assume that Fix $(T) \neq \emptyset$. Then for all $x$ in $C,\left(T^{n} x\right) \Delta$-converges to a fixed point of $T$.

Proof. Since $\operatorname{Fix}(T) \neq \emptyset$, we get that $r_{C}(T)=0$, so, by Corollary [5.2, we get that $T$ is asymptotically regular. Now apply Proposition 6.3.

6.1. An application to a minimization problem. Let $(X, d)$ be a complete CAT(0) space and $F: X \rightarrow(-\infty, \infty]$ be a proper, convex and lower semicontinuous mapping. We shall apply Theorem 6.4 to approximate the minimizers of $F$, that is, the solutions of the minimization problem $\min _{x \in X} F(x)$.

Let $\underset{y \in X}{\operatorname{argmin}} F(y)=\{x \in X \mid F(x) \leq F(y)$ for all $y \in X\}$ be the set of minimizers of $F$. The following result is a consequence of the definition of the resolvent and Proposition 2.13 .

Proposition 6.5. For all $\mu>0$, the set Fix $\left(J_{\mu}\right)$ of fixed points of the resolvent associated with $F$ coincides with the set $\underset{y \in X}{\operatorname{argmin}} F(y)$ of minimizers of $F$.

Proof. Let $\mu>0$.

" $\supseteq$ " If $\bar{x}$ is a minimizer of $F$, one gets that $\mu F(\bar{x}) \leq \mu F(y)+d(\bar{x}, y)^{2}$. It follows that $\bar{x} \in \underset{y \in X}{\operatorname{argmin}}\left\{\mu F(y)+d(\bar{x}, y)^{2}\right\}$. By the definition of $J_{\mu}$, it follows that $J_{\mu}(\bar{x})=\bar{x}$. 
"ᄃ" Assume that $J_{\mu}(\bar{x})=\bar{x}$, so $\mu F(\bar{x}) \leq \mu F(y)+d(\bar{x}, y)^{2}$ for all $y \in X$. Let $\gamma:[0, c] \rightarrow X$ be a geodesic starting with $\bar{x}$. Then for all $t \in[0, c]$, one has that

$$
\frac{F(\gamma(t))-F(\bar{x})}{t} \geq-\frac{d(\bar{x}, \gamma(t))^{2}}{\mu t}=-\frac{t^{2}}{\mu} .
$$

It follows that $D_{\gamma} F(\bar{x}) \geq 0$, hence we can apply Proposition 2.13 to conclude that $F(\bar{x}) \leq F(y)$ for all $y \in X$.

As the resolvent is a firmly nonexpansive mapping, one can apply Theorem 6.4 and the above result to obtain

Corollary 6.6. Assume that $F$ has a minimizer. Then for all $\mu>0$ and all $x \in X$, the Picard iterate $\left(J_{\mu}^{n}(x)\right) \Delta$-converges to a minimizer of $F$.

We remark that a more general result was recently obtained by Bačák 1] using different methods. Thus, Bačák obtained in the setting of $\operatorname{CAT}(0)$ spaces the following proximal point algorithm: if $F$ has minimizers, then for all $x_{0} \in X$ and all sequences $\left(\lambda_{n}\right)$ divergent in sum, the sequence

$$
x_{n+1}:=\underset{y \in X}{\operatorname{argmin}}\left(F(y)+\frac{1}{2 \lambda_{n}} d\left(y, x_{n}\right)^{2}\right)
$$

$\Delta$-converges to a minimizer of $F$.

\section{EFFECTIVE RATES OF ASYMPtotic REgularity}

As we have proved in Section 5, any $\lambda$-firmly nonexpansive mapping $T: C \rightarrow C$ defined on a nonempty subset $C$ of a $W$-hyperbolic space $X$ is asymptotically regular, provided $T$ has bounded orbits.

In this section we shall obtain, for $U C W$-hyperbolic spaces, a rate of asymptotic regularity of $T$, that is, a rate of convergence of the sequence $\left(d\left(T^{n} x, T^{n+1}\right)\right)$ towards 0 . The methods of proof are inspired by those used by Kohlenbach [23] and the second author [29] for computing rates of asymptotic regularity for the Krasnoselski-Mann iterations of nonexpansive mappings in uniformly convex Banach spaces and $U C W$-hyperbolic spaces.

For $x \in C$ and $b, \varepsilon>0$, let us denote

$$
\operatorname{Fix}_{\varepsilon}(T, x, b):=\{y \in C \mid d(y, x) \leq b \text { and } d(y, T y)<\varepsilon\} .
$$

If $\operatorname{Fix}_{\varepsilon}(T, x, b) \neq \emptyset$ for all $\varepsilon>0$, we say that $T$ has approximate fixed points in a $b$-neighborhood of $x$.

Theorem 7.1. Let $b>0, \lambda \in(0,1)$ and $\eta:(0, \infty) \times(0,2] \rightarrow(0,1]$ be a mapping that decreases with $r$ for fixed $\varepsilon$. Then for all $U C W$-hyperbolic spaces $(X, d, W, \eta)$, nonempty subsets $C \subseteq X, \lambda$-firmly nonexpansive mappings $T: C \rightarrow C$ and all $x \in C$ such that $T$ has approximate fixed points in a b-neighborhood of $x$, the following holds:

$$
\forall \varepsilon>0 \forall n \geq \Phi(\varepsilon, \eta, \lambda, b)\left(d\left(T^{n} x, T^{n+1} x\right) \leq \varepsilon\right),
$$


where

$$
\Phi(\varepsilon, \eta, \lambda, b):= \begin{cases}{\left[\frac{b+1}{\varepsilon \lambda(1-\lambda) \eta\left(b+1, \frac{\varepsilon}{b+1}\right)}\right]} & \text { for } \varepsilon<2 b, \\ 0 & \text { otherwise. }\end{cases}
$$

Remark 7.2. If, moreover, $\eta(r, \varepsilon)$ can be written as $\eta(r, \varepsilon)=\varepsilon \cdot \tilde{\eta}(r, \varepsilon)$ such that $\tilde{\eta}$ increases with $\varepsilon$ (for a fixed $r$ ), then the bound $\Phi(\varepsilon, \eta, \lambda, b)$ can be replaced for $\varepsilon<2 b$ by

$$
\tilde{\Phi}(\varepsilon, \eta, \lambda, b)=\left[\frac{b+1}{\varepsilon \lambda(1-\lambda) \tilde{\eta}\left(b+1, \frac{\varepsilon}{b+1}\right)}\right] .
$$

Before proving the above results, let us give two consequences.

Corollary 7.3. Let $b, \lambda, \eta$ be as in the hypothesis of Theorem 7.1, Then for all $U C W$-hyperbolic spaces $(X, d, W, \eta)$, bounded subsets $C \subseteq X$ with diameter $d_{C} \leq b$, $\lambda$-firmly nonexpansive mappings $T: C \rightarrow C$ and all $x \in C$,

$$
\forall \varepsilon>0 \forall n \geq \Phi(\varepsilon, \eta, \lambda, b)\left(d\left(T^{n} x, T^{n+1} x\right) \leq \varepsilon\right),
$$

where $\Phi(\varepsilon, \eta, \lambda, b)$ is given by (30).

Proof. If $C$ is bounded, then $T$ is asymptotically regular by Corollary 5.3. Hence, for all $b \geq d_{C}, T$ has approximate fixed points in a $b$-neighborhood of $x$ for all $x \in C$.

Thus, for bounded $C$, we get that $T$ is asymptotically regular with a rate $\Phi(\varepsilon, \eta, \lambda, b)$ that only depends on $\varepsilon$, on $X$ via the monotone modulus of uniform convexity $\eta$, on $C$ via an upper bound $b$ on its diameter $d_{C}$ and on the mapping $T$ via $\lambda$. The rate of asymptotic regularity is uniform in the starting point $x \in C$ of the iteration and other data related with $X, C$ and $T$.

As we have remarked in Section 2. CAT(0) spaces are $U C W$-hyperbolic spaces with a quadratic (in $\varepsilon$ ) modulus of uniform convexity $\eta(\varepsilon)=\frac{\varepsilon^{2}}{8}$, which has the form required in Remark 7.2. As an immediate consequence, we get a quadratic (in $1 / \varepsilon)$ rate of asymptotic regularity in the case of $\mathrm{CAT}(0)$ spaces.

Corollary 7.4. Let $b>0$ and $\lambda \in(0,1)$. Then for all $C A T(0)$ spaces $X$, bounded subsets $C \subseteq X$ with diameter $d_{C} \leq b, \lambda$-firmly nonexpansive mappings $T: C \rightarrow C$ and $x \in C$, the following holds:

$$
\forall \varepsilon>0 \forall n \geq \Psi(\varepsilon, \lambda, b)\left(d\left(T^{n} x, T^{n+1} x\right) \leq \varepsilon\right),
$$

where

$$
\Psi(\varepsilon, \lambda, b):= \begin{cases}{\left[\frac{8(b+1)}{\lambda(1-\lambda)} \cdot \frac{1}{\varepsilon^{2}}\right]} & \text { for } \varepsilon<2 b \\ 0 & \text { otherwise }\end{cases}
$$


7.1. Proof of Theorem $\mathbf{7 . 1}$ and Remark 7.2. One can easily see that $d\left(T^{n} x\right.$, $\left.T^{n+1} x\right) \leq 2 b$ for all $n \in \mathbb{N}$; hence the case $\varepsilon \geq 2 b$ follows.

Assume now that $\varepsilon<2 b$ and denote

$$
N:=\Phi(\varepsilon, \eta, \lambda, b)=\left[\frac{b+1}{\varepsilon \lambda(1-\lambda) \eta\left(b+1, \frac{\varepsilon}{b+1}\right)}\right] .
$$

Let $\delta>0$ be such that $\delta<\frac{1}{4(N+1)}$, so that $(N+1) \delta<\frac{1}{4}<1$. By hypothesis, there exists $y \in C$ satisfying

$$
d(x, y) \leq b \quad \text { and } \quad d(y, T y)<\delta .
$$

We shall prove that there exists $n \leq N$ such that $d\left(T^{n} x, T^{n+1} x\right) \leq \varepsilon$ and apply the fact that $\left(d\left(T^{n} x, T^{n+1} x\right)\right)$ is nonincreasing to get the conclusion. Assume by contradiction that $d\left(T^{n} x, T^{n+1} x\right)>\varepsilon$ for all $n=0, \ldots, N$. In the sequel, we fix such an $n$. For simplicity we shall use the notation

$$
r_{n}:=d\left(T^{n} x, y\right)+d(y, T y) .
$$

One gets by an easy induction that

$$
r_{n} \leq d(x, y)+(n+1) d(y, T y) \leq b+(N+1) \delta<b+1 .
$$

Since

$$
\begin{gathered}
d\left(T^{n+1} x, y\right) \leq d\left(T^{n+1} x, T y\right)+d(T y, y) \leq r_{n}, \quad d\left(T^{n} x, y\right) \leq r_{n}, \\
d\left(T^{n} x, T y\right) \leq d\left(T^{n} x, y\right)+d(y, T y)=r_{n}, \quad d\left(T^{n+1} x, T y\right) \leq r_{n}
\end{gathered}
$$

and $d\left(T^{n} x, T^{n+1} x\right)>\varepsilon$, we can twice apply Lemma 2.10(iii) with $r:=r_{n}$ and $s:=b+1$ to get that

$$
\begin{aligned}
d\left((1-\lambda) T^{n} x \oplus \lambda T^{n+1} x, y\right) & \leq\left(1-2 \lambda(1-\lambda) \eta\left(b+1, \frac{\varepsilon}{b+1}\right)\right) r_{n}, \\
d\left((1-\lambda) T^{n} x \oplus \lambda T^{n+1} x, T y\right) & \leq\left(1-2 \lambda(1-\lambda) \eta\left(b+1, \frac{\varepsilon}{b+1}\right)\right) r_{n} .
\end{aligned}
$$

As $T$ is $\lambda$-firmly nonexpansive, it follows that

$$
\begin{aligned}
d\left(T^{n+1} x, T y\right) \leq & d\left((1-\lambda) T^{n} x \oplus \lambda T^{n+1} x,(1-\lambda) y \oplus \lambda T y\right) \\
\leq & (1-\lambda) d\left((1-\lambda) T^{n} x \oplus \lambda T^{n+1} x, y\right) \\
& +\lambda d\left((1-\lambda) T^{n} x \oplus \lambda T^{n+1} x, T y\right) \quad \text { by }(\mathrm{W} 1) \\
\leq & \left(1-2 \lambda(1-\lambda) \eta\left(b+1, \frac{\varepsilon}{b+1}\right)\right) r_{n} \\
= & d\left(T^{n} x, y\right)+d(y, T y)-2 r_{n} \lambda(1-\lambda) \eta\left(b+1, \frac{\varepsilon}{b+1}\right) \\
\leq & d\left(T^{n} x, y\right)+\delta-\varepsilon \lambda(1-\lambda) \eta\left(b+1, \frac{\varepsilon}{b+1}\right),
\end{aligned}
$$

since $d(y, T y) \leq \delta$ and

$$
\frac{\varepsilon}{2}<\frac{1}{2} d\left(T^{n} x, T^{n+1} x\right) \leq \frac{1}{2}\left(d\left(T^{n} x, y\right)+d(y, T y)+d\left(T y, T^{n+1} x\right)\right) \leq r_{n} .
$$


Now using the fact that $d\left(T^{n+1} x, y\right) \leq d\left(T^{n+1} x, T y\right)+d(y, T y)$, we get that

$$
d\left(T^{n+1} x, y\right) \leq d\left(T^{n} x, y\right)+2 \delta-\varepsilon \lambda(1-\lambda) \eta\left(b+1, \frac{\varepsilon}{b+1}\right) .
$$

Adding (35) for $n=0, \ldots, N$, it follows that

$$
\begin{aligned}
d\left(T^{N+1} x, y\right) & \leq d(x, y)+2(N+1) \delta-(N+1) \varepsilon \lambda(1-\lambda) \eta\left(b+1, \frac{\varepsilon}{b+1}\right) \\
& \leq b+\frac{1}{2}-(N+1) \varepsilon \lambda(1-\lambda) \eta\left(b+1, \frac{\varepsilon}{b+1}\right) \\
& \leq b+\frac{1}{2}-(b+1)<0,
\end{aligned}
$$

a contradiction.

To prove Remark 7.2 observe that by denoting

$$
N:=\tilde{\Phi}(\varepsilon, \eta, \lambda, b)=\left[\frac{b+1}{\varepsilon \lambda(1-\lambda) \tilde{\eta}\left(b+1, \frac{\varepsilon}{b+1}\right)}\right]
$$

and following the proof above with $r_{n}$ instead of $b+1$, we obtain

$$
\begin{aligned}
d\left(T^{n+1} x, T y\right) \leq & d\left(T^{n} x, y\right)+\delta-r_{n} \lambda(1-\lambda) \eta\left(r_{n}, \frac{\varepsilon}{r_{n}}\right) \\
\leq & d\left(T^{n} x, y\right)+\delta-r_{n} \lambda(1-\lambda) \eta\left(b+1, \frac{\varepsilon}{r_{n}}\right) \\
& \quad \text { since } \eta \text { is monotone } \\
= & d\left(T^{n} x, y\right)+\delta-\varepsilon \lambda(1-\lambda) \tilde{\eta}\left(b+1, \frac{\varepsilon}{r_{n}}\right) \\
\leq & d\left(T^{n} x, y\right)+\delta-\varepsilon \lambda(1-\lambda) \tilde{\eta}\left(b+1, \frac{\varepsilon}{b+1}\right)
\end{aligned}
$$

since $\tilde{\eta}$ increases with $\varepsilon$.

Now follow the proof above to get the conclusion.

\section{ACKNOWLEDGEMENTS}

The first author was supported by Junta de Andalucia, Grant FQM-3543. Part of his research was carried out while visiting the Simion Stoilow Institute of Mathematics of the Romanian Academy.

The second author was supported by a grant of the Romanian National Authority for Scientific Research, CNCS - UEFISCDI, project number PN-II-ID-PCE-20113-0383.

The third author was partially supported by DGES, Grant MTM2009-13997C02-01 and Junta de Andalucia, Grant FQM-127.

The authors gratefully acknowledge the anonymous reviewer for helpful comments and suggestions. 


\section{REFERENCES}

[1] M. Bačák, The proximal point algorithm in metric spaces, Israel J. Math. 194 (2013), no. 2, 689-701. DOI 10.1007/s11856-012-0091-3. MR3047087

[2] Heinz H. Bauschke, Sarah M. Moffat, and Xianfu Wang, Firmly nonexpansive mappings and maximally monotone operators: correspondence and duality, Set-Valued Var. Anal. 20 (2012), no. 1, 131-153, DOI 10.1007/s11228-011-0187-7. MR2886508 (2012m:47082)

[3] H. Brezis, M. G. Crandall, and A. Pazy, Perturbations of nonlinear maximal monotone sets in Banach space, Comm. Pure Appl. Math. 23 (1970), 123-144. MR0257805 (41 \#2454)

[4] Martin R. Bridson and André Haefliger, Metric spaces of non-positive curvature, Grundlehren der Mathematischen Wissenschaften [Fundamental Principles of Mathematical Sciences], vol. 319, Springer-Verlag, Berlin, 1999. MR1744486 (2000k:53038)

[5] Felix E. Browder, Convergence theorems for sequences of nonlinear operators in Banach spaces, Math. Z. 100 (1967), 201-225. MR0215141 (35 \#5984)

[6] Ronald E. Bruck Jr., Nonexpansive projections on subsets of Banach spaces, Pacific J. Math. 47 (1973), 341-355. MR0341223 (49 \#5973)

[7] Ronald E. Bruck and Simeon Reich, Nonexpansive projections and resolvents of accretive operators in Banach spaces, Houston J. Math. 3 (1977), no. 4, 459-470. MR0470761 (57 \#10507)

[8] M. Bruhat and J. Tits, Groupes réductifs sur un corps local. I. Données radicielles valuées, Inst. Hautes Études Sci. Publ. Math. 41 (1972), 5-251. MR0327923

[9] Herbert Busemann, Spaces with non-positive curvature, Acta Math. 80 (1948), 259-310. MR.0029531 (10,623g)

[10] Herbert Busemann, The geometry of geodesics, Academic Press Inc., New York, N.Y., 1955. MR0075623(17,779a)

[11] Michael Edelstein, The construction of an asymptotic center with a fixed-point property, Bull. Amer. Math. Soc. 78 (1972), 206-208. MR0291917 (45 \#1005)

[12] Michael Edelstein, Fixed point theorems in uniformly convex Banach spaces, Proc. Amer. Math. Soc. 44 (1974), 369-374. MR0358451 (50 \#10917)

[13] Rafa Espínola and Aurora Fernández-León, CAT $(k)$-spaces, weak convergence and fixed points, J. Math. Anal. Appl. 353 (2009), no. 1, 410-427, DOI 10.1016/j.jmaa.2008.12.015. MR2508878 (2010d:47092)

[14] M. Fekete, Uber die Verteilung der Wurzeln bei gewissen algebraischen Gleichungen mit ganzzahligen Koeffizienten (German), Math. Z. 17 (1923), no. 1, 228-249, DOI 10.1007/BF01504345. MR1544613

[15] O. P. Ferreira, L. R. Lucambio Pérez, and S. Z. Németh, Singularities of monotone vector fields and an extragradient-type algorithm, J. Global Optim. 31 (2005), no. 1, 133-151, DOI 10.1007/s10898-003-3780-y. MR2141129(2006b:58009)

[16] Jesús García-Falset and Simeon Reich, Zeroes of accretive operators and the asymptotic behavior of nonlinear semigroups, Houston J. Math. 32 (2006), no. 4, 1197-1225 (electronic). MR2268479 (2007m:47156)

[17] Kazimierz Goebel and W. A. Kirk, Iteration processes for nonexpansive mappings, Topological methods in nonlinear functional analysis (Toronto, Ont., 1982), Contemp. Math., vol. 21, Amer. Math. Soc., Providence, RI, 1983, pp. 115-123, DOI 10.1090/conm/021/729507. MR729507 (85a:47059)

[18] Kazimierz Goebel and Simeon Reich, Uniform convexity, hyperbolic geometry, and nonexpansive mappings, Monographs and Textbooks in Pure and Applied Mathematics, vol. 83, Marcel Dekker Inc., New York, 1984. MR744194 (86d:58012)

[19] Jürgen Jost, Equilibrium maps between metric spaces, Calc. Var. Partial Differential Equations 2 (1994), no. 2, 173-204, DOI 10.1007/BF01191341. MR1385525 (98a:58049)

[20] Jürgen Jost, Convex functionals and generalized harmonic maps into spaces of nonpositive curvature, Comment. Math. Helv. 70 (1995), no. 4, 659-673, DOI 10.1007/BF02566027. MR.1360608 (96j:58043)

[21] W. A. Kirk, Krasnosel'skiu's iteration process in hyperbolic space, Numer. Funct. Anal. Optim. 4 (1981/82), no. 4, 371-381, DOI 10.1080/01630568208816123. MR673318(84e:47067)

[22] W. A. Kirk and B. Panyanak, A concept of convergence in geodesic spaces, Nonlinear Anal. 68 (2008), no. 12, 3689-3696, DOI 10.1016/j.na.2007.04.011. MR2416076 (2009m:54061) 
[23] Ulrich Kohlenbach, Uniform asymptotic regularity for Mann iterates, J. Math. Anal. Appl. 279 (2003), no. 2, 531-544, DOI 10.1016/S0022-247X(03)00028-3. MR1974043 (2004d:47098)

[24] Ulrich Kohlenbach, Some logical metatheorems with applications in functional analysis, Trans. Amer. Math. Soc. 357 (2005), no. 1, 89-128 (electronic), DOI 10.1090/S0002-994704-03515-9. MR2098088(2005h:03110)

[25] U. Kohlenbach, Applied proof theory: proof interpretations and their use in mathematics, Springer Monographs in Mathematics, Springer-Verlag, Berlin, 2008. MR2445721 (2009k:03003)

[26] U. Kohlenbach and L. Leuştean, Asymptotically nonexpansive mappings in uniformly convex hyperbolic spaces, J. Eur. Math. Soc. (JEMS) 12 (2010), no. 1, 71-92, DOI 10.4171/JEMS/190. MR2578604 (2011d:47112)

[27] Eva Kopecká and Simeon Reich, Asymptotic behavior of resolvents of coaccretive operators in the Hilbert ball, Nonlinear Anal. 70 (2009), no. 9, 3187-3194, DOI 10.1016/j.na.2008.04.023. MR2503064(2010d:47095)

[28] Tadeusz Kuczumow, An almost convergence and its applications (English, with Russian and Polish summaries), Ann. Univ. Mariae Curie-Skłodowska Sect. A 32 (1978), 79-88 (1980). MR687863(84f:47066)

[29] L. Leustean, A quadratic rate of asymptotic regularity for CAT(0)-spaces, J. Math. Anal. Appl. 325 (2007), no. 1, 386-399, DOI 10.1016/j.jmaa.2006.01.081. MR2273533 (2008a:54048)

[30] Laurenţiu Leuştean, Nonexpansive iterations in uniformly convex $W$-hyperbolic spaces, Nonlinear analysis and optimization I. Nonlinear analysis, Contemp. Math., vol. 513, Amer. Math. Soc., Providence, RI, 2010, pp. 193-210, DOI 10.1090/conm/513/10084. MR2668247 (2011k:47099)

[31] Chong Li, Genaro López, and Victoria Martín-Márquez, Monotone vector fields and the proximal point algorithm on Hadamard manifolds, J. Lond. Math. Soc. (2) 79 (2009), no. 3, 663-683, DOI 10.1112/jlms/jdn087. MR2506692 (2010e:47167)

[32] Teck Cheong Lim, Remarks on some fixed point theorems, Proc. Amer. Math. Soc. 60 (1976), 179-182 (1977). MR0423139 (54 \#11120)

[33] Uwe F. Mayer, Gradient flows on nonpositively curved metric spaces and harmonic maps, Comm. Anal. Geom. 6 (1998), no. 2, 199-253. MR.1651416 (99m:58067)

[34] George J. Minty, Monotone (nonlinear) operators in Hilbert space, Duke Math. J. 29 (1962), 341-346. MR0169064 (29 \#6319)

[35] Jean-Jacques Moreau, Proximité et dualité dans un espace hilbertien (French), Bull. Soc. Math. France 93 (1965), 273-299. MR0201952 (34 \#1829)

[36] Olavi Nevanlinna and Simeon Reich, Strong convergence of contraction semigroups and of iterative methods for accretive operators in Banach spaces, Israel J. Math. 32 (1979), no. 1, 44-58, DOI 10.1007/BF02761184. MR531600 (80e:47057)

[37] A. Nicolae, Asymptotic behavior of averaged and firmly nonexpansive mappings in geodesic spaces, Nonlinear Anal. 87 (2013), 102-115. MR3057039

[38] Athanase Papadopoulos, Metric spaces, convexity and nonpositive curvature, IRMA Lectures in Mathematics and Theoretical Physics, vol. 6, European Mathematical Society (EMS), Zürich, 2005. MR2132506 (2005k:53042)

[39] Simeon Reich, Extension problems for accretive sets in Banach spaces, J. Functional Analysis 26 (1977), no. 4, 378-395. MR0477893 (57 \#17393)

[40] Simeon Reich and Itai Shafrir, The asymptotic behavior of firmly nonexpansive mappings, Proc. Amer. Math. Soc. 101 (1987), no. 2, 246-250, DOI 10.2307/2045990. MR.902536 (88i:47030)

[41] Simeon Reich and Itai Shafrir, Nonexpansive iterations in hyperbolic spaces, Nonlinear Anal. 15 (1990), no. 6, 537-558, DOI 10.1016/0362-546X(90)90058-O. MR1072312 (91k:47135)

[42] R. Tyrrell Rockafellar, Monotone operators and the proximal point algorithm, SIAM J. Control Optimization 14 (1976), no. 5, 877-898. MR0410483 (53 \#14232)

[43] Ryszard Smarzewski, On firmly nonexpansive mappings, Proc. Amer. Math. Soc. 113 (1991), no. 3, 723-725, DOI 10.2307/2048607. MR1050023 (92b:47089)

[44] E. N. Sosov, On analogues of weak convergence in a special metric space (Russian), Izv. Vyssh. Uchebn. Zaved. Mat. 5 (2004), 84-89; English transl., Russian Math. (Iz. VUZ) 48 (2004), no. 5, 79-83. MR2101682(2005f:54008) 
[45] I. Stojkovic, Geometric approach to evolution problems in metric spaces, Ph.D. thesis, 2011, http://www.math.leidenuniv.nl/scripties/PhDThesisStojkovic.pdf.

[46] Wataru Takahashi, A convexity in metric space and nonexpansive mappings. I, Kōdai Math. Sem. Rep. 22 (1970), 142-149. MR0267565 (42 \#2467)

[47] Hong-Kun Xu, Strong asymptotic behavior of almost-orbits of nonlinear semigroups, Nonlinear Anal. 46 (2001), no. 1, Ser. A: Theory Methods, 135-151, DOI 10.1016/S0362546X(99)00453-8. MR1845582(2002g:47135)

Departamento Análisis Matemático, Fac. Matemáticas, Universidad de Sevilla, Apdo. 1160, 41080-SEvilla, Spain

E-mail address: dariza@us.es

Simion Stoilow Institute of Mathematics of the Romanian Academy, P. O. Box 1-764, RO-014700 Bucharest, Romania

E-mail address: Laurentiu.Leustean@imar.ro

Departamento Análisis Matemático, Fac. Matemáticas, Universidad de Sevilla, Apdo. 1160, 41080-SEvilla, Spain

E-mail address: glopez@us.es 